\title{
The Changing Epidemiology of Extended Spectrum Beta-Lactamases (ESBL) Infections of the Urinary Tract Focusing on Clinical Resistance and Therapeutic Options
}

\author{
Suresh J. Antony \\ Texas Tech University Medical Center/Paul Foster School of Medicine \\ and The Center for Infectious Diseases and Travel Medicine \\ El Paso, Texas \\ USA
}

\section{Introduction}

The first beta- lactamases were identified in a species of E.coli in 1940 (1). However, the ability of bacteria to produce enzymes that destroy the b-lactam ring was noted even before penicillin was developed. In fact, many of the gram-negative bacteria possess chromosomally mediated b- lactamases, which help the bacteria find a niche when faced with competition from other bacteria that naturally produce b-lactams.

In 1965, the first plasmid mediated beta- lactamases was discovered. This occurred in a strain of E.coli isolated from the blood culture of a patient from Greece whose name was Temoniera. The beta -lactamases was named TEM-1 after the patient's name from whom it was isolated. (2) This strain soon spread to other members of the Enterobacteriaceae species, Hemophilus influenza, Neisseria gonorrhoeae and Pseudomonas aeruginosa due to the plasmid mediated transfer.

Around the same time a second plasmid mediated beta-lactamases was found in Klebsiella pneumonaie and E.coli. This was called SHV-1 (sulfhydryl variable) (3). The advent of the blactam class of antibiotics was influenced largely by the discovery of these enzymes. An example of this was the development of oxyimino-cephalosporin, which showed good stability against the TEM-1 and SHV-1 b-lactamases (4). This class of antibiotics soon became the workhorse for these types of serious infections.

Unfortunately, resistance to this class soon became evident in 1985 with beta-lactamases showing the ability to hydrolyze these compounds in K.pneumoniae (5). Because this enzyme was noted to be active against expanded spectrum b-lactams these enzymes were labeled as "extended spectrum beta-lactamases" -ESBL.

Several b-lactamases have continued to be with over 130 TEMS types and over 50 SHV types known to date. These are mainly found in E.coli, K.pneumoniae and P.mirabilis, but have also been found in other species of the Enterobacteriacae family and even in some nonenteric bacteria such as Acinetobacter species.

Shortly after the introduction of new broad-spectrum cephalosporins such as cefotaxime and ceftazidime, non-TEM and non SHV ESBL's were discovered. This new class of ESBL's 
has been called CTX-M in reference to the potent hydrolytic activity of these enzymes against cefotaxime(6). There are over 40 of these enzymes reported. CTX-M producing ESBL pathogens usually have cefotaxime in the resistant range (MIC>64).

More recently, and of greater concern is the occurrence of carbapenemases which show activity against oxyimino-cephalosporins and cephamycins but also against carbapenems (7). There are two major groups in this class called metallo-b-lactamases (Verona integron encoded metallo b lactamases) (VIM) and carbapenemases. Structural studies of ESBL indicate that active site expansion and remodeling are responsible for the extended hydrolytic activity (8).These enzymes are globally present and appear to cause clinically significant disease such as urinary tract infections, abscesses and bacteremia.

With the advent of the ESBL pathogens, there has been a significant increase in the morbidity and mortality related to these infections. If the number of carbapenemaseproducing organisms continues to increase, the treatment options will be seriously compromised.

In addition, ESBL producing pathogens are not only resistant to penicillin and cephalosporins but also to trimethoprim-sulphamethoxazole and fluroquinolones which can compromise the treatment of both nosocomial and community acquired infections caused by Enterobacteriaceae and other species (9).

One of the major clinical problems has been the recognition of both nosocomial and community acquired urinary tract infections resulting from ESBL pathogens. The treatment options for these infections are limited, especially in the out patient setting.

This chapter will review the epidemiology, risk factors, clinical features and therapeutics options for ESBL-induced infections of the urinary tract.

\section{Recent epidemiological data}

ESBL producing organisms have been implicated in nosocomial infections. Over the last decade, there has been a steady increase of these infections in the community.

In fact, a recent study from Spain suggest there was been an increase in ESBL E.coli producers from $0.3 \%$ to $4.8 \%$ between 1995 and 2002. (10) Interestingly, during this same period there was a drop in the rate of ESBL producing K.pneumoniae following the control of nosocomial transmission of this pathogen. These K. pneumoniaie were mostly clonally related and produced SHV and TEM.

In contrast, the ESBL E.coli strains were not clonally related and the predominant strain was a CTX-M. In addition, half of these strains were isolated from outpatients $(10,11)$.

France was one of the first countries to report an outbreak of ESBL infections in 1986. In this study, 30\% of Enterobacter aerogenes isolates in 2000 were ESBL producers (12). Since that time, virtually every country in Europe has reported ESBL producers with considerable geographical variability in the occurrence of ESBL's. Examples of this include a prevalence rate of ESBLs

K. pneumoniaie in Sweden of 3\% to 34\% in Portugal (13). In one study done in France, it was noted that intestinal carriage prevalence of ESBL-E.coli was $8.0 \%$, mainly the CTX-M type. At the same time, it was noted that there was an increase in antibiotic usage, especially the beta-lactams. This variability probably occurred because of the repeated introduction of new strains and plasmids and from inter-individual dissemination (14)

In Central and South America ESBL, rates in Klebsiella varied from 30 to $60 \%$ in countries such as Brazil, Columbia and Venezuela (15). The ESBL strains included SHV-2, 5, CTX-M and even non-TEM and non-SHV with no geographical predilection. $(16,17)$ 
In Africa and the Middle East there has been a number of outbreaks of ESBL producing infections from South Africa to northern Africa. The rates of ESBL were variable depending on the country (18 19).

In North America the first case of an ESBL producer was in 1988and since then a variety of infections produced by TEM strains, SHV type and CTM-X have been reported. In fact, in a recent survey it was noted that non-susceptibility to third generation cephalosporin's may be as high as $13 \% .(20,21)$. In the outpatient, setting $1.8 \%$ of k.pneumoniae and $0.4 \%$ were ceftazidime resistant (22)

In Asia, there seems to be a larger proportion of ESBL pathogens. Studies from several countries, including China, India, Japan, Korea, and Malaysia showed ranges from $30 \%$ to $40 \%(23,24)$. Reports of a possible predominant CTM-X ESBL in countries like India, China Korea, Japan and Taiwan indicate that there may be a dominant ESBL type in Asia $(25,26)$. More recently, there have been studies showing increasing numbers of carbapenemase producing pathogens, which is of increasing clinical importance due to the lack of effective antimicrobial therapy. (27-31).

Current data suggest that the incidence of ESBL producing infections is on the rise globally resulting in increasing difficulty in the diagnosis and treatment of these infections.

\section{In vitro resistance studies for ESBL}

In-vitro susceptibility testing of cephalosporins for ESBL producing enterobacteriacae can be misleading. Testing may suggest that an isolated strain is susceptible to a given cephalosporin, but the drug may not be effective when used to treat a serious infection caused by the organism. Thus, CLSI guidelines recommend that laboratories report ESBL producing isolates as resistant to all penicillin's, cephalosporins and aztreonam irrespective of in vitro results. (32).

In vitro studies performed in Turkey found that E.coli isolated from CA-UTI infections had simultaneous resistance to trimethoprim-sulphamethaxazole, ciprofloxacin, and gentamicin in $4.6 \%$ of an ESBL negative group and $39.2 \%$ in the ESBL positive group. $90 \%$ of these ESBL isolates were found to have CTX-M 15. (33). This data is worrisome as therapeutic options are limited when oral antibiotics are used.

In Taiwan, Lau and colleagues looked at 201 patients with and without bacteremia in CAESBL UTI. They found that e.coli was the most common pathogen and was more frequent in the bacteremic than non-bacteremic group. Non-E.coli isolates such as K.pneumoniae, Morganella morganii etc were more common in the non-bacteremic group. E.coli isolates had a high rate of resistance to ampicillin (80\%), gentamicin (29\%) trimethoprimsulphamethaxazole (56\%). (34). Similar findings have been documented from other parts of the world such as Saudi Arabia (35).

Detection of ESBL's is based on the fact that ESBL producers should be reported as resistant to all penicillin's, cephalosporins (except cephamycins) and aztreonam irrespective of routine antimicrobial susceptibility testing. (32)

Both broth dilution and disk diffusion can be used for the screening of ESBL producers. Specific phenotypic confirmatory tests should be done if the E.coli, K.pneumoniae, show MIC's $>8 \mathrm{ug} / \mathrm{ml}$ for cefpodoxime or $\mathrm{MIC}^{\prime} \mathrm{s}>2 \mathrm{ug} / \mathrm{ml}$ against ceftazidime, cefotaxime or aztreonam. $(36,37)$

The E-test can also be used in the detection of ESBL. Automated methods for bacterial identification and susceptibility testing are also used in the detection of ESBL producing 
organisms. These include the BD Phoenix system, Vitek 2 system and the Micoscan Walkway -96 system.

\section{Risk factors for colonization /infection with ESBL}

There have been several case controlled studies looking at the risk factors for colonization with or without infection due to ESBL producers. However, the results are conflicting due to study populations, geographical areas, selection of cases and controls and sample size. (3848).

Despite these statistical differences, some generalizations can be made. (Table 1)

\begin{tabular}{|l|}
\hline Diabetes mellitus \\
\hline Previous antimicrobial exposure (quinolones, third generation cephalosporins, penicillin) \\
\hline Previous hospital admissions \\
\hline Older age \\
\hline Male patients \\
\hline
\end{tabular}

Table 1. Risk factors for the Development of Community ESBL Infections.

Some of these risk factors include seriously ill patients with prolonged hospital stays (11-67 days) who have usually had multiple invasive devices and co-morbidities such as urinary catheters, central lines, nasogastric tubes, jejunostomy tubes, arterial lines, total parental administration, recent surgery, decubitus ulcers, hemodialysis catheters and poor nutritional status.

The use of previous antibiotics such as third generation cephalosporins, quinolones, trimethoprim-sulphamethoxazole, aminoglycosides and metronidazole have also been implicated in several studies. $(38,42,45,49,44,50,47,48,51)$.

\section{Community -acquired infections involving ESBL pathogens}

In a large French study in 1993, looking at E.coli, K.pneumoniae and P .mirabilis (2500 isolates) from non-hospitalized patients, there was no evidence of community acquired ESBL infections (52). Since then, there have been several studies of true community acquired ESBL infections. These involved patients with diarrheal diseases such as Shigella, Salmonella, Vibrio cholerae and E. coli. (53-56)

The prevalence of colonization with enterobacteria is unknown. The percentage of ESBL producing Enterobacteria faecal carriers in Spain increased from $2.1 \%$ to $7.5 \%$ IN 2002. (57) . The most frequent types of ESBL were CTX-M, followed by SHV. In India, the rate of fecal carriage was $7 \%$ in a sample of healthy adults (60). In Canada, Pitout found 5.5 cases per 100,000 populations with $69 \%$ being community acquired. (59)

Three case controlled studies looking at risk factors for ESBL E.coli outpatient infections found that diabetes mellitus, previous use of antibiotics such as quinolones and cephalosporins, recurrent urinary tract infections, prior hospital admissions and older age were independent risk factors $(59,60)$. However, infections due to ESBL producing E.coli in patients can occur without obvious risk factors. This may be related to the increase in healthy carriers colonized with this pathogen.

Colodoner et al evaluated 128 cases of UTI caused by ESBL E.coli and K. pneumoniae and found that age $>60$ years, male sex, previous use of quinolones or cephalosporins, previous 
hospitalization, and previous infections caused by K. pneumoniae were independent risk factors.(61)

In community- acquired urinary tract infection (CA-UTI), the rate of ESBL associated UTI's varied from $1.4 \%$ in Spain up to $3.3 \%$ in the Gaza strip $(60,62,63)$.

In the last 7 years, there have been an increasing number of publications from several countries showing and increase of community acquired ESBL infections, mainly in urinary tract. $(64,65,61,57,30,59,60,31)$ Most of these patients had urinary tract infections (UTI's) with genes encoding for CTM-X type of ESBL's (60). Recently, there has been an increase in the diagnosis of infections caused by ESBL E.coli producers diagnosed in the outpatient's setting. $(59,60,30)$

Romero et al showed an increase from $0.3 \%$ in 1995 to $4.8 \%$ in 2002 in community acquired ESBL E.coli producers. (10). These ESBL E.coli producing strains were not clonally related with the majority belonging to the CTX-M family and more than $50 \%$ were isolated in the outpatient setting. $(10,11)$

In one study in Spain up to $6.5 \%$ of community, acquired bacteremia was associated with ESBL E.coli UTI (60).

In summary, ESBL infections can range from colonization to carriage to true infections involving sepsis syndromes and bacteremia.

\section{Clinical features of CA-UTI infections caused by ESBL pathogens}

Several studies have described the microbiological features of ESBL producing organisms in the outpatient setting. However, very few studies have correlated the microbiological findings with that of the clinical features and prognosis of these CA-UTI ESBL infections. Therefore, one may only draw some tentative conclusions from these studies.

In urinary tract infections, the majority of ESBL's isolated, not surprisingly, have been ESBL E.coli. This organism has also been isolated from other sources such as wounds, sputum, and occasionally blood. $(59,60,11$,

In the United States, Chao Qi et al evaluated 193 single patient ESBL isolates in outpatient urine cultures during a 5-year period. $3 \%$ of E.coli had ESBL and this was noted to have increased 14 times from 2003 to 2008. This increase may have been in part due to the dissemination of CTX-M type of ESBL. (66) This was also noted in another study from nursing homes and out patient clinics. (67) . Resistance to ciprofloxacin and trimethoprimsulphamethaxazole was much higher as well.

In another study of 49 patients with ESBL E.coli infections, ESBL E.coli was isolated from urine in 47 of the cases and from blood in 6 of the patients. Thirty-seven (76\%) of these patients were considered to have symptomatic infections and $11(22 \%)$ asymptomatic bactiuria. 1 patient also had cholangitis and $6(13.5 \%)$ of these patients were bacteremic. In this same study, 10 of the 28 patients who received antibiotics actually received an appropriate agent to which the organism was susceptible in vitro. $13 \%$ of these patients had a UTI relapse. There were no deaths in this study (60). It appears that the complication rate with CA-ESBL UTI's may not be higher than that associated with routine non-ESBL pathogens, although further studies are still needed. The main predictor of mortality caused by ESBL E.coli is probably inadequate initial antimicrobial therapy. In comparison, ESBL-EC associated mortality for hospitalized patients with serous infections such as bacteremia and sepsis was about $25 \%-31 \%$. This was also associated with inadequate empiric antibiotic therapy. $(68,69)$. 
The most frequent cause of community-acquired bacteremia is E.coli $(70,71)$ and currently available antibiotics such as quinolones, beta-lactams and third generation cephalosporins are commonly used to treat them. This may need to change with the advent of increasing antimicrobial resistance and increasing mortality associated with these CA-ESBL infections $(44,59)$. Rodriguez-Bano examined CA-ESBL associated bacteremia and its features and found that in 95 patients with blood stream infections $7.3 \%$ were due to E.coli. The majority belonged to the CTX-M family of ESBL and was clonally unrelated. The risk factors associated with these patients included urinary foley catheter use and previous antimicrobial exposure (60). The sources of bacteremia were the urinary tract, intraabdominal sites and respiratory tract. Interestingly, mortality associated with blood stream infections due to ESBL-E.coli was lower among patients who received empirical therapy with beta-lactam or it combinations or carbapenems than among those that received quinolones. In addition, higher mortality was associated with inappropriate empirical therapy in patients with bacteremia due to E.coli. (72)

In patients with solid organ transplants and renal transplants, the major site of infection was the urinary tract in $72 \%$ of the cases, with ESBL .K. pneumoniae being more common in renal transplant patients ( 73).

Geriatric patients with ESBL UTI's pose an unusual clinical problem. These patients may be chronically colonized in either the gastrointestinal tract or the skin and reinfection is a possibility. In addition, many of these patients are asymptomatic and do not present with the classic symptoms of dysuria, frequency of urination, fever or leukocytosis. In general, one may not need to treat asymptomatic ESBL infections. If there is a change in the clinical status such as fever, leukocytosis or altered mental status then treatment options should be considered. Numerous outbreaks have been reported of patients with ESBL infections. Much of the spread is plasmid mediated and is therefore through direct and indirect transmission. Contact isolation should be instituted in patients with ESBL infections.

In summary, ESBL infections can present from simple colonization to active UTI's and to serious bacteremia associated with sepsis syndrome.

\section{Treatment options for ESBL UTI infections}

Treatment options for ESBL infections are the same for both nosocomial and community acquired infections. The major problem at this time is the lack of effective oral antibiotics for the treatment of outpatient ESBL infections.

\subsection{Overview of available antibiotics}

ESBL's hydrolyze aztreonam, penicillin and cephalosporins (with the exception of cephamycins) with varying degrees of hydrolytic activity. Usually the TEM and SHV type ESBL's have greater hydrolytic activity for ceftazidime than for cefotaxime (74). Therefore, ESBL producing organisms may appear susceptible to some of the above-mentioned antibiotics in vitro. In addition, there is frequent co -expression of resistance by these organisms to classes of antimicrobial agents other than those hydrolyzed by the ESBL's. This has been documented for quinolones, aminoglycosides, tetracycline's (excluding glycylcycline) and trimethoprim-sulphamethoxazole (59)

Some of the other antibiotic classes used to treat ESBL infections include beta lactam/betalactamases inhibitors. The level of activity for these agents varies by the type of inhibitor 
and by the class of ESBL. For example, tazobactam appears to be more effective than clavulanic acid against certain types of CTX-M type ESBL's and both of these agents are more effective than sulbactam in inhibiting TEM and SHV type ESBL. $(75,76)$. This data is mainly from in-vitro studies. Clinical information is sparse in regards to beta-lactam and inhibitor combinations, but some favorable outcomes have been reported with pipercillin/tazobactam. However, it is important to note that favorable results have not been consistently reported $(74,77)$. One possible oral option may be amoxicillin/clavulanate, which has shown some activity in CA-ESBL Enterobacteriaceae UTI infections $(60,78)$

Few studies have evaluated cephalosporins in the treatment of both bacteremic and nonbacteremic ESBL infections. The results have been equivocal when ceftazidime or cefepeme were compared to Imipenem in $e$. coli bacteremia and in ICU patients with Enterobacteriaceae infections. $(79,80)$. In vitro data also suggests suboptimal outcomes when the cephalosporins were used to treat ESBL infections. Thus, most experts' advise against using cephalosporins in the treatment of ESBL associated infections. $(79,80)$

Cephamycins have not been well studied in the treatment of ESBL associated infections. In one small retrospective study, there was no obvious difference in the mortality rates between the cephamycins and carbapenems. Recent studies have documented resistance to the cephamycins $(49,74)$.

The glycylcycline class of antibiotics, specifically tigecycline, thus far evaded the common mechanisms of resistance in both gram positive and gram-negative pathogens. It has excellent in vitro activity against ESBL-E.coli and K. pneumoniae. However, clinical data is sparse in the treatment of ESBL UTI's and bacteremia. In addition, only a fraction of the drug is excreted in the urine as unchanged drug. In addition, tigecycline does not achieve high concentrations in the blood, casting doubts on its potential effectiveness in the treatment of bacteremia.(81)

Fosfomycin has been used in Europe but is not available in most parts of the world. It is a phosphor derivative of streptomycin and inhibits cell wall synthesis and impairs adherence to urogenital mucosa. A study in Spain found that the resistance rate to fosfomycin of ESBL-EC was $0.3 \%$. (82). It has been used in cystitis and asymptomatic UTI in pregnancy. $(82,83)$. In the United States $90 \%$ of the isolates in one study were susceptible to fosfomycin and to a combination of cefdinir plus amoxicillin-clavulanate. $(84,85)$

Pivmecillinam is a beta lactam antibiotic, which binds penicillin-binding protein 2 (PBP-2) and inhibits cell wall synthesis. This drug has been used in the treatment of cystitis due to Enterobacteriaceae. (86).

Nitrofurantoin is a bactericidal drug, which acts by altering bacterial ribosome's proteins and can be used for UTI as well.

Finally, carbapenems are considered the drug of choice for ESBL infections. All the drugs in the class appear to have the same efficiency in the treatment of ESBL. Ertapenem, is the only drug in this class that can be administered once a day. It can be used in the outpatient setting as long as the in vitro activity is similar to imipenem, doripenem or meropenem. $(87,88)$. However, recent reports of carbapenem resistance have emerged and the spread of resistance is of concern. One possible option might be to add amikacin to the empiric regimen in community-acquired sepsis originating in the urinary tract since amikacin resistance among CTX-M isolates is relatively low.

The treatment for upper UTI's may have to be limited to the intravenous antibiotics mentioned above especially as the patients tend to be sicker and may present with systemic 
inflammatory response syndrome and occasionally bacteremia. These should include carbapenems. Occasionally, ampicillan-sulbactam and tigecycline may be alternate therapies although data on these drugs in the treatment of ESBL UTI infections is sparse.

In lower UTI's, some of the oral antibiotics such as nitrofurantoin, fosphomycin, amoxicillinclavulanate and trimethoprim-sulphamethaxazole may be used if the pathogen is susceptible to them.

\section{Conclusion}

Antimicrobial resistance has become a global problem of increasing importance. It is now essential that laboratories be able to rapidly identify and characterize resistant organisms. This is, of even more importance, in ESBL producing organisms that clearly have a higher morbidity and mortality associated with their infections. There is also increasing evidence, that ESBL organisms frequently possess resistance factors to other classes of other antimicrobials, like the aminoglycosides and quinolones. ESBL producing bacteria are being found both in the hospital and in the community, especially the CTX-M beta -lactamases.

The increasing number of community isolates, especially E. coli producing CTX-M-15 have become global and now are being seen in the hospital as well. It is thought that the CTX-M 15 producing E.coli is mostly due to a single clone named ST131, which appears to have originated in the Indian sub-continent. In addition, the increasing number of carbapenemases could also seriously compromise our treatment options. Therefore, empiric antimicrobial coverage may need to be modified in patients who present with serious sepsis syndromes, especially, after travel to countries that are high risk for this clone.

Treatment of ESBL infections requires the use of carbapenems in seriously ill patients. Imipenem, meropenem, doripenem are all viable alternatives. Ertapenem can be used in the out patient setting, in the absence of Pseudomonas aeruginosa. Agents such as fosfomycin, nitrofurantoin, amoxicillin/clavulanic acid, pivemecillinam, temocillin can be alternate drugs in uncomplicated UTI's and in patients with drug allergies to the carbapenems. Salvage therapy using tigecycline and colistin can be used in seriously ill patients who are CTX-M producers and Amp-C producing isolates.

In addition to understanding the complex mechanisms involved in ESBL infections, strict antimicrobial stewardship, appropriate infection control measures and aggressive treatment of seriously ill patients is necessary in reducing the mortality and morbidity associated with these infections.

\section{References}

[1] Abraham EP, Chain E(1940): An enzyme from bacteria able to destroy penicillin (letter). Nature ; $146: 837$

[2] Datta N, Kontomichalau P(1965): Penicillinase synthesis controlled by infectious R factors in Enterobacteriaceae. Nature ;208:239-44

[3] Pitton JS (1972): Mechanism of bacterial resistance to antibiotics. Rev. Physiol Biochem Pharmacol.;65:15-93

[4] Neu HC (1982). The new beta-lactamase stable cephalosporins. Ann Intern Med ;97:40819

[5] Kiebe C et al (1985): Evolution of plasmid coded resistance to broad-spectrum cephalosporins. Antimicrobial Agents chemotherar .28:302-7 
[6] Bonnet R (2004). Growing extended spectrum of beta lactamases: the CTX-M enzymes.Antimicrob Agents Chemother;48:1-14

[7] Nordmann P, Poirel L (2002): Emerging carbapenemases in gram-negative aerobes. Clin Microbiol Infect;8:321-31

[8] Perez F et al (2007): The continuing challenges of ESBL'S. Curr Opin Pharmacol oct 7(5):459-469.

[9] Paterson DL et al (2001). Outcome of cephalosporins treatment for serious infections due to apparently susceptibility organism producing extended spectrum beta lactamases: implications for the clinical microbiology laboratory. J Clin Microbiol;39:2206-12

[10] Romero L et al (2005) A: long-term study of the frequency of ESBL producing e.coli and Klebsiella pneumonia isolates. Clin Microbiol Infect. 11;625-631

[11] Hernandez JR et al (2005) A. Spanish Group for nosocomial infections . Nationwide study of Escheria coli and Klebsiella pneumoniae producing extended spectrum beta lactamases in Spain. Antimicrobial Agents Chemother. ;49;2122-2125

[12] Albertini Mt et al (2002). Surveillance of methicillin resistant staphylococcus aureus and enterobacteriacae producing extended spectrum beta lactamases (ESBL) in Northern France. A 5-year multicentre study. . J hosp Infec;52:107-113.

[13] Handberger $\mathrm{H}$ et al(1999): Antibiotic susceptibility among gram negative bacilli in intensive care units in 5 European countries. French and Portuguese ICU study groups. .JAMA 281:67-71

[14] Woerther PL et al (2010). Emergence and dissemination of extended spectrum beta lactamases producing Escherichia coli in the community: lessons from the study of a remote and controlled population. J Infect Dis. Aug 15;(4):515-23

[15] Mendes C et al (2000): evaluation of the in vitro activity of 9 antimicrobials against bacterial strains isolated from patients in the intensive care units in Brazil. MYSTIC antimicrobial surveillance Program.2000. Braz J Infect Dis. 4:236-244

[16] Casellas JM, Quinteros MG . A Latin American point de vu on the epidemiology, control and treatment options of infections caused by extended spectrum betalactamase producers. In Anabile-cuevas CF. editor. Antimicrobial resistance in bacteria. Wymondham Horizon bioscience. 99-122

[17] Bauernfeind A et al (1992). A new plasmidic cefotaximase from patients infected with salmonella typhimurium . Infection .20:158-163

[18] Aitmhand R et al (2002). Plasmid mediated TEM 3 extended spectrum beta lactamase production in salmonella typhimurium in Casablanca. J Antimicrobial Chemother.49;169-179

[19] Barguellil $\mathrm{F}$ et al (1995). In vitro acquisition of extended spectrum beta lactamases in salmonella enteritis during antimicrobial therapy. Eur j Clin Microbiol Infect Dis. 14:703-706

[20] Neuwirth CS et al (2001). TEM 89 beta lactamase produced by a Proteus mirabilis clinical isolate. New complex mutant with mutations in both TEM 59 and TEM 3. Antimicrobial Agents Chemother 45:3591-3594

[21] Myers KS et al (1993): Nosocomial outbreak of Klebsiella infection resistant late generation cephalosporin's. . Ann Intern Med. 119:353-358 
[22] National Nosocomial Infections Surveillance, 2002 nation nosocomial Infections Surveillance system report, data summary from January 1992 to June 2002, issued august 2002. Am J Infect Control.30:458-475

[23] Bell JM et al (2007). Prevalence and significance of a negative extended spectrum beta lactamase confirmation test result after a positive ESBL screening test result for isolates of Escherichia coli and Klebsiella pneumonaie : result form the Sentry Asia pacific surveillance program. J Antimicrobial Chemother.;45:1478-82

[24] Yu WLet al (2002). Molecular epidemiology of extended spectrum beta lactamase producing fluroquinolones resistant isolates of Klebsiella pneumoniae in Taiwan. J Clin Microbiol.;40:4666-9

[25] Chanawong A et al (2002). Three cefotaximases, CTX-M-9, CTX-M-13 and CTX-M-14 among enterobacteriacae in the People Republic of China. Antimicrobial Agents Chemither.;46;630-637

[26] Karim A, Poirel S, Nagarajan S, Nordmann P (2001): Plasmid mediated extended spectrum beta lactamase (CTX-M like)from India and gene association with insertion sequence ISEcpL. .FEMS Microbiol Lett.201:237-241

[27] Koh TH et al (2001). Carbapenem resistant Klebsiella pneumoniae in Singapore producing IMP-1 beta lactamases and lacking an outer membrane protein. Antimicrobial agents Chemother. ;45:1939-40

[28] Miriagou V, Tassos PT, Legakis NJ, Tzouvelckis LS (2004). Extended spectrum cephalosporin resistance in non-typhoid salmonella. Int. J Antimicrobial Agents;23:547-55

[29] Lincopan NJ et al (2005). First isolation of metallo beta lactamase producing multiresistent Klebsiella pneumoniae from a patient in Brazil. J clin Microbiol. 25:516-519

[30] Munday CJ et al(2004). Predominance and genetic diversity of community and hospital acquired CTX-M extended spectrum beta -lactamases in York, UK. J Antimicrobial Chemother.;54:628-33

[31] Woodford N et al (2007). Molecular epidemiology of multi resistant Escherichia coli isolates from community onset urinary tract infections in Cornwall, England. J Antimicrob Chemother.59(1)106-109

[32] Clinical and laboratories Standards Institute. Performance standards for antimicrobial susceptibility testing. fifteen Informational supplement. M100-S15, CSI, Wayne PA, USA 2005

[33] Azap OK et al (2010).Risk factors for extended spectrum beta lactamases positivity in uropathogenic Escherichia coli isolated from community acquired urinary tract infections. Clin Microbiol Infect Feb;16 (2): 147-51.

[34] Lau SM, Peng MY, Chang FY (2004). Resistance rates to community used antimicrobials among pathogens of both bacteremic and non-bacteremic community acquired urinary tract infections. J Microbiol Immunol Infect.. Jun ;37(3):185-91

[35] Khanfar $\mathrm{H}$ et al (2009). Extended spectrum beta lactamases in Escheria coli and Klebsiella pneumoniae: trends in hospital and community settings. J Infect Dev Ctries. 3(4) 295-299

[36] Carter MW et al (2000): Detection of extended spectrum beta lactamases in Klebsiella with the oxford combination disk method. J Clin Microbiol .38:4228-4232 
[37] Weigand I et al (2007). Detection of extended spectrum beta lactamases in Enterobacteriaceae by use of semi automated microbiology systems and manuel detection procedures. . j clin Microbiol .45;1167-1174

[38] Canton R, Coque TM (2006): The CTX-M beta lactamases pandemic. Curr opion Microbiol..9:466-476

[39] Arsenio A et al (2000). Outbreak of a multidrug resistant Klebsiella pneumonaie strain in an intensive care unit: antibiotic use as a risk factor for colonization and infection. . Clin Infect Dis. 30> 55-60

[40] Bisson G et al (2002). Extended spectrum beta lactamase producing E. coli and klebsiella species : risk factors for colonization's and impact of antimicrobial formulary interventions on colonization's prevalence. Infect Control Hosp Epidemiol. 23:254260

[41] DAgata E et al (1998). The molecular and clinical epidemiology of enterobacteriacae producing extended spectrum beta lactamases in a tertiary care hospital. 36. 279285.

[42] Lautenbach E et al (2001), Strom BL, Bilker WB, Patel JB, Edelstein PH, Fishman NO. epidemiological investigation of fluroquinolones resistance in infections due to extended spectrum beta lactamase producing Escherichia coli and klebsiella pneumoniae . Clin Infect Dis.;33:1288-94

[43] Mangeney N et al ( ) G. A 5-year epidemiological study of extended spectrum beta lactamases producing klebsiella pneumoniae isolates

[44] Peterson LP (2008). Antimicrobial policy and prescribing strategies for therapy of extended spectrum beta lactamases producing enterobacteriacae: the role of pipercillin tazobactam . Clin Microbiol Infect.14(suppl)181-184

[45] Pena c et al (2008). Infections due to Escherichia coli producing extended spectrum beta lactamase among hospitalized patients: factors influencing mortality. J Hosp Infect. Feb ;68(2):116-22.

[46] Helfand MS, Bonomo RA (2006): Extended spectrum beta lactamases in multidrug resistant Escherichia coli . Changing the therapy for hospital acquired and community acquired infections. Clin Infect Dis.;43:1415-1416

[47] Schiappa D et al (1996), Hayden MK, Matushek MG, Hasemi FM, Sullivan J, Smith KY, Miyashiro D, Quinn JP, Weinstein RA, Trenholme GM. Ceftazidime resistant klebsiella pneumonaie and Escheria coli blood stream infection . a case control and epidemiological investigation. J Infect Dis. 174:529-536

[48] Weiner J et al (1999). Multiple antibiotic resistant Klebsiella and E coli in nursing homes.. JAMA 281:517-523

[49] Lee CH, Su LH, Tang YF, Liu JW (2006). Treatment of ESBL producing Klebsiella pneumoniae bacteremia with carbapenems or flomofef: a retrospective study and laboratory analysis of the isolates. J Antimicrob Chemother.;58:1074-1077.

[50] Silva J, Aguillar C, Becerra Z et al (1999), Lopez Antunano F, Garcia R: extended spectrum beta lactamases in clinical isolated of enterobactericae in Mexico.. Microb drug Resist. 5:189-193

[51] DeChamps C et al (1991), Rouby D, Guelon D, Sirot J, Sirot D, Beytout D, Gourgand JM. A case controlled study of an infections caused by Klebsiella pneumoniae strains producing CTX-M (TEM-3) beta lactamases. . J Hosp Infect. 18:5-13 
[52] Goldstein FW, Pean Y, Gartner J (1995): Resistance to ceftriaxone and other beta lactam in bacteria isolated in the community. The Vigil Roc study group. 1995. Antimicrob Agents Chemother.. 39:2516-2519

[53] Baranniak A et al (2002), Sadowy E, Hryniewicz W, Gniadkowski M: Two different extended spectrum beta lactamase(ESBL) in one of the first ESBL producing salmonella isolates in Poland.. J Clin Microbiol.. 40:1095-1097

[54] Fortineau N, Naas T, Gaillot O, Nordmann P (2001). SHV type extended spectrum beta lactamase in a Shigella flexerni clinical isolates. J Antimicrob Chemother.. 47:685688

[55] Kim S et al (2004). Occurrence of extended spectrum beta lactamases in members of the genus Shigella in the republic of Korea.. J clin Microbiol. 42:5264-5269

[56] Ishil Y et al(1995). Cloning and sequence of the gene encoding a cefotaxime hydrolyzing class A beta lactamase isolated from E coli.. Antimicrob Agents Chemother. 39;2269-2275

[57] Mirelis B et al(2003), Navarro F, Miro E, Mesa RJ, Coll P, Prats G. Community transmission of extended spectrum beta lactamases. Emerg Infect Dis 9;1024-1025

[58] Rodriguez Bano J, Navarro MD (2008). Extended spectrum beta lactamases in ambulatory care: a clinical perspective. Clin Microbiol Infect.. Jan ;14 Suppl 1:104-10

[59] Pitout J et al (2004), Hanson N, Church DL, Laupland KB. Population based laboratory surveillance for Escheria coli producing extended spectrum beta lactamases: importance of community isolates with the bla CtX-m genes. Clin Infec Dis. 38;1736-1741.

[60] Rodriguez Bano J, Alcala JC, Cisneros JM et al (2008). Community infections caused by extended spectrum beta lactamases producing Escheria coli. Arch intern med..168:1897-1902.

[61] Colodner R, Rock W, Chazan B (2004). Risk factors for the development of extended spectrum beta lactamase producing bacteremia in hospitalized patients. Eur J Clin Microbiol Infect Dis..23:163-7

[62] Calbo E et al (2006 J. risk factors for community onset urinary tract infections due to Escheria coli harboring extended spectrum beta lactamases. J Antimicrob. Chemother.. 57; 780-783.

[63] Astal Z, Sharif SA, Abdallah SA et al (2004). Extended spectrum beta lactamases in escheria Coli isolated from community acquired urinary tract infections in the Gaza Strip.. Ann Saudi Med. 24:55-57

[64] Bloomberg B et al (2005). High rate of pediatric septicemia caused by gram-negative bacteremia with extended spectrum beta lactamases in Dar-es- Salam, Tanzania. J Clin Microbiol. 43:745-749fatal cases

[65] Brigante GF et al ( ). Evolution of CTX-M type beta lactamases in isolates of escheria coli infecting hospital and

[66] Chao Qi et al (2010),. Changing prevalence of Escherichia coli with CTX-M type extended spectrum beta lactamases in out patient urinary e.coli between 2003-2008. Diag Microbiol and Infect Dis.. 67:87-91

[67] Hanson ND (2003). Amp C beta lactamases: what do we need to know for the future.?. J Antimicrob Chemother. 52.2-4

[68] Ramphal R, Ambrose P (2006): Extended spectrum beta lactamases and clinical outcomes. Clin Infect Dis;42:S164-72 
[69] Kang CL et al (2008), Cheong HS, Chung DR, Peck KP, Song JH, Oh MD, Choe KW. Clinical features and outcome of community onset bloodstream infections caused by extended spectrum beta lactamase producing Escherichia coli. . Eur J Clin Microbiol Infect Dis. Jan; 27(1)85-8

[70] Wisplinghoff $\mathrm{H}$ et al (2003). Nosocomial blood stream infections in US Hospitals: analysis of 24,179 cases from a prospective nationwide study. Clin Infect Dis.;39:309-317

[71] Friedman R, Raveh R, Zartzer E, et al (2009).Prospective evaluation of colonization with extended spectrum beta lactamase producing enterobacteriacae among patients at hospital admission and of subsequent colonization with ESBL producing enterobactericae among patients during hospitalization.. Infect Control Hosp Epidemiol. 30:534-542

[72] Kim Y et al (2002), Pai H, Lee HJ, Park SE, Choi EH, Kim J, Kim JH, Kim EC. Blood stream infections by extended spectrum beta lactamases producing escheria coli and klebsiella pneumoniae in children. Epidemiology and clinical outcomes.. Antmicrob Agents Chemother.46:1481-1491.

[73] Cervera C et al (2010), linares L, Hoyo G, Sanclemente G, Marco F, Cofan F, Coftan F, Ricart MJ, Navasa M, Moreno A: Klebsiella pneumoniae infection in solid organ transplant: Epidemiology and antibiotic resistance. Transplantation Proceeding, 42, 2941-2943.

[74] Paterson DL. Bonomo RA (2005). Extended spectrum beta lactamases. C clinical update. Clin Microbiol rev.18;657-686

[75] Bush K et al (1993), Macanthil C, Rasmussen BA, Lee VJ et al.Kinetic interactions of tazobactam with beta lactamases from all major structural classes. Antimicrob Agents Chemother.;37:851-858

[76] Payne DJ et al(1994): comparative activities of clavulanic acid, sulbactam and tazobactam against clinically important beta lactamases. Antimicrob Agents Chemother.;38:767-772

[77] Burgess DS et al (2003). Clinical and microbiologic analysis of a hospitals extended spectrum beta lactamases producing isolates over a two-year period. Pharmacotherapy. ;23:1232-1237

[78] Falagas ME, Polemis M, Alexiou VG et al (2008). Antimicrobial resistance of escheria e.coli isolates from primary care patients in Greece. Med sci Monit;14:CR75-CR79.

[79] Bin C, Hui W, Renyuan Z et al (2006): outcome of cephalosporin treatment of bacteremia due to CTX-M type extended spectrum beta lactamases producing Escherichia coli. Diag Microbiol Infec Dis.;56:351-357.

[80] Goethaert K, Van Looveran M, Lammens C et al: High does cefepeme as an alternative treatment for infections caused by TEM-24 ESBL producing Enterobacter aerogenes in severely ill patients. Clin Microbiol Infect 2006;12:56-62

[81] Morosini MI et al(2006): Antibiotic co resistance in extended spectrum beta lactamases producing enterobacteriacae and in vitro activity of tigecycline. Antimicrob Agents Chemother:50:2695-2699

[82] Garau J (2008). other antimicrobials of interest in the era of extended spectrum beta lactamases: fosfomycin, nitrofurantoin, and tigecycline. Clin Microbiol Infect.14(suppl). P198-202 
[83] Auer S, Wojna A, Hell M (2010): Oral treatment options for ambulatory patients with urinary tract infections caused by extended spectrum beta lactamases producing e.coli. Antimicrobial Agents Chemother. Sept .p4006-4008

[84] Prakash V et al (2009), Lewis JS, Herrara ML, Wickes BL, Jorgenson JH: oral and parental therapeutic options for out patient urinary infections caused by enterobacteriacae producing CTX-M extended spectrum beta-lactamase. Antimicrob Agents Chemotherar. March;53(3):1278-80

[85] Depersio JR et al (2005): Evolution and dissemination of extended spectrum beta lactamases producing Klebsiella pneumoniae: Epidemiology and molecular report from the Sentry Antimicrobial surveillance program. (1997-2003).Diag Microbiol infec Dis . 51(1):1-7

[86] Zahar JR, Lortholary O, Martin C et al: Addressing the challenge of extended spectrum beta lactamases. Curr Opion Investig.Drugs 2009;Feb 10.(2):172-80

[87] Hernandez JR et al (2006), Velasco C, Romero C, Martinez,-Martinez L, Pascual A. A comparative in-vitro activity of ertapenem against extended spectrum beta lactamase producing escheria coli and klebsiella pneumoniae isolated in Spain.. Int Antimicrob Agents. 28:457-9.2006

[88] Livermore DM, Oakton KJ carter Mw et al (2001), Warner M: Activity of ertapenem (MK-0826) versus Enterobacteriaceae with potent beta lactamases. Antimicrobial. Agents Chemother 45(10): 2831-2837 


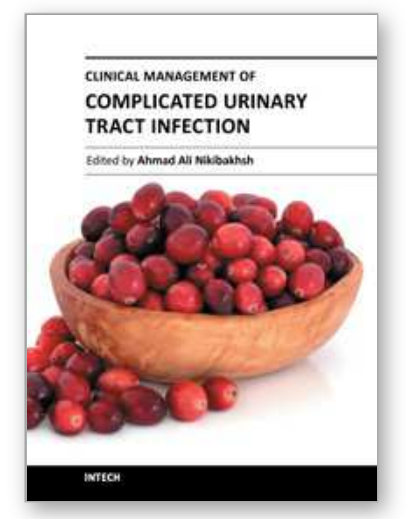

\author{
Clinical Management of Complicated Urinary Tract Infection \\ Edited by Dr. Ahmad Nikibakhsh
}

ISBN 978-953-307-393-4

Hard cover, 294 pages

Publisher InTech

Published online 06, September, 2011

Published in print edition September, 2011

Complicated urinary tract infections (cUTIs) are a major cause of hospital admissions and are associated with significant morbidity and health care costs. Knowledge of baseline risk of urinary tract infection can help clinicians make informed diagnostic and therapeutic decisions. Prevalence rates of UTI vary by age, gender, race, and other predisposing risk factors. In this regard, this book provides comprehensive information on etiology, epidemiology, immunology, pathology, pathogenic mechanisms, symptomatology, investigation and management of urinary tract infection. Chapters cover common problems in urinary tract infection and put emphasis on the importance of making a correct clinical decision and choosing the appropriate therapeutic approach. Topics are organized to address all of the major complicated conditions frequently seen in urinary tract infection. The authors have paid particular attention to urological problems like the outcome of patients with vesicoureteric reflux, the factors affecting renal scarring, obstructive uropathy, voiding dysfunction and catheter associated problems. This book will be indispensable for all professionals involved in the medical care of patients with urinary tract infection.

\title{
How to reference
}

In order to correctly reference this scholarly work, feel free to copy and paste the following:

Suresh J. Antony (2011). The Changing Epidemiology of Extended Spectrum Beta-Lactamases (ESBL) Infections of the Urinary Tract Focusing on Clinical Resistance and Therapeutic Options, Clinical Management of Complicated Urinary Tract Infection, Dr. Ahmad Nikibakhsh (Ed.), ISBN: 978-953-307-393-4, InTech, Available from: http://www.intechopen.com/books/clinical-management-of-complicated-urinary-tractinfection/the-changing-epidemiology-of-extended-spectrum-beta-lactamases-esbl-infections-of-the-urinarytract-

\section{INTECH}

open science | open minds

\author{
InTech Europe \\ University Campus STeP Ri \\ Slavka Krautzeka 83/A \\ 51000 Rijeka, Croatia \\ Phone: +385 (51) 770447 \\ Fax: +385 (51) 686166 \\ www.intechopen.com
}

\author{
InTech China \\ Unit 405, Office Block, Hotel Equatorial Shanghai \\ No.65, Yan An Road (West), Shanghai, 200040, China \\ 中国上海市延安西路65号上海国际贵都大饭店办公楼 405 单元 \\ Phone: +86-21-62489820 \\ Fax: +86-21-62489821
}


(C) 2011 The Author(s). Licensee IntechOpen. This chapter is distributed under the terms of the Creative Commons Attribution-NonCommercialShareAlike-3.0 License, which permits use, distribution and reproduction for non-commercial purposes, provided the original is properly cited and derivative works building on this content are distributed under the same license. 